\title{
A note on *complete intersections generated by linear forms
}

\author{
M. La Barbiera
}




\title{
A NOTE ON *COMPLETE INTERSECTIONS GENERATED BY LINEAR FORMS
}

\author{
M. LA BARBIERA
}

Received 18 September, 2012

\begin{abstract}
Let $R$ be a commutative noetherian graded ring. In $R\left[Y_{1}, \ldots, Y_{m}\right]$ we consider the linear forms $a_{i}=\sum_{j=1}^{m} a_{j i} Y_{j}, 1 \leq i \leq n$, with $a_{j i}$ homogeneous elements of $R$. We state a necessary and sufficient condition, in terms of * grades of the determinantal ideals of the matrix $A=\left(a_{j i}\right)$, for the ideal $\left(a_{1}, \ldots, a_{n}\right)$ to be a * complete intersection of ${ }^{*}$ grade $n$ in $R\left[Y_{1}, \ldots, Y_{m}\right]$.

2010 Mathematics Subject Classification: 13A02; 13A15; 13C99
\end{abstract}

Keywords: graded rings, linear forms, complete intersections

\section{INTRODUCTION}

Let $R$ be a commutative noetherian ring with unit. In [1] the author considered relation ideals of symmetric algebras of finitely generated $R$-modules and stated a condition to be a complete intersection. If $R$ is a graded ring, similar properties can be established and studied. Definitions and results are inspired by the results known in the not graded case, however it is interesting to establish them in the graduate case. In fact, for the graded algebras good properties can be determined, as in the local case ([2-4]).

Let $E$ be a finitely generated $R$-module and $\operatorname{Sym}_{R}(E)=\bigoplus_{t=0}^{\infty} S y m_{t}(E)$ be its symmetric algebra. It is known that the symmetric algebra $\operatorname{Sym}_{R}(E)$ has a presentation $R\left[Y_{1}, \ldots, Y_{m}\right] / J$, where $m$ is the number of the generators of $E$ and $J$ is the relation ideal generated by the linear forms $a_{i}=\sum_{j=1}^{m} a_{j i} Y_{j}, 1 \leq i \leq n$ in the variables $Y_{1}, \ldots, Y_{m}$. Theoretical properties of $\operatorname{Sym}_{R}(E)$ (such as integrity, regularity, to be Cohen-Macaulay, Gorenstein) were studied by various authors. For a finitely generated module $E$ this topic developed and culminated in the study of approximation complex of $E$. For best results the projective resolution of $E$ can be examined, when it is finite. In particular, Avramov studied theoretic properties of $\operatorname{Sym}_{R}(E)$ in terms of a condition on Fitting ideals of $E$, when the projective dimension of $E$ is $\leq 1$, and stated a condition for the presentation ideal of $\operatorname{Sym}_{R}(E)$ to be a complete intersection.

The aim of this note is to investigate such properties in the graduate case. 
The paper is organized as follows. In Section 1 some preliminary notions and properties on graded rings are introduced. Let $R$ be a graded ring and $I$ be an arbitrary ideal of $R . I^{*}$ is the graded ideal generated by all homogeneous elements of $I$ and it is the largest graded ideal contained in $I$. In particular, for each prime ideal $\wp \subset R$, the graded ideal $\wp^{*}$ is a prime ideal too. In [2] there are theorems that link theoretic properties of $\wp$ and $\wp^{*}$ by localizations of finitely generated graded $R$-modules, that is $\operatorname{dim} E_{\wp}=\operatorname{dim} E_{\wp}+1$ and depth $E_{\wp}=\operatorname{depth} E_{\wp} *+1$, for all not graded prime ideal $\wp$ of $R$. Now, we generalize the classic definitions for graded ideals. We define * grade of $I$ the common length of the maximal homogeneous $R$-sequences in $I^{*}$. Then $I$ is a ${ }^{*}$ complete intersection if it can be generated by a number of homogeneous elements equal to its * grade. In Section 2 we consider homogeneous linear forms $a_{i}=\sum_{j=1}^{m} a_{j i} Y_{j}, 1 \leq i \leq n$, of $R\left[Y_{1}, \ldots, Y_{m}\right]$ with $a_{j i}$ homogeneous elements of $R$. We state a necessary and sufficient condition, in terms of * grades of the determinantal ideals of the matrix $A=\left(a_{j i}\right)$, such that $\left(a_{1}, \ldots, a_{n}\right)$ is a ${ }^{*}$ complete intersection of * grade $n$ in $R\left[Y_{1}, \ldots, Y_{m}\right]$.

\section{PReliminary and notations}

Let $R$ be a commutative noetherian graded ring. In [2] there are some definitions related to the graded ideals of $R$ and some properties on the dimension and the depth of some localizations of finitely generated graded $R$-modules.

Definition 1. Let $I \subset R$ be an ideal (not necessarily graded). $I^{*}$ is the graded ideal of $R$ generated by all the homogeneous elements of $I$ (the homogeneous elements of $I$ of degree $j$ are $f \in I_{j}=I \cap R_{j}$ ).

The ideal $I^{*}$ is the largest graded ideal contained in $I$. If $I$ is homogeneous, then $I=I^{*}$.

Remark 1. Let $\wp \subset R$ be a prime ideal, then $\wp^{*}$ is a prime ideal too ([2], 1.5.6).

Example 1. Let $I=\left(X_{1}^{2}-2, X_{1} X_{2}-2\right) \subset R=K\left[X_{1}, X_{2}\right]$. We have:

$$
\begin{aligned}
& I_{0}=I \cap R_{0}=(0) \\
& I_{1}=I \cap R_{1}=(0) \\
I_{2}= & I \cap R_{2}=\left\langle X_{1}^{2}-X_{1} X_{2}\right\rangle,
\end{aligned}
$$

in fact $f=X_{1}^{2}-2-\left(X_{1} X_{2}-2\right)=X_{1}^{2}-X_{1} X_{2} \in I_{2}$. In general, for $i>2$ :

$$
I_{i}=I \cap R_{i}=\left\langle g\left(X_{1}^{2}-X_{1} X_{2}\right)\right\rangle,
$$

with $\operatorname{deg}(g)=i-2$. It follows that $I^{*}=\left(X_{1}^{2}-X_{1} X_{2}\right) \subset I$.

We recall the following well-known property ([2]).

Proposition 1. Let $R$ be a commutative noetherian graded domain and $I=(f) \subset$ $R$ be a principal ideal. $I^{*}=(0) \Longleftrightarrow f$ is not a homogeneous element. 
The previous proposition is not true if $R$ is not a domain as the following example shows.

Example 2. Let $R={ }_{8}[X]$ be the graded ring with standard graduation and $I$ be the ideal of $R$ generated by the not homogeneous element $f=X-4$. In $I$ there are homogeneous elements, hence $I^{*} \neq(0)$. In fact, let $a=2 X \in R_{1}$ be a homogeneous element of $R$, we have $a f=2 X^{2} \in I$ that is a homogeneous element of degree two. Hence $2 X^{2} \in I^{*}$.

Remark 2. Let $R$ be a graded ring that is not a domain and $\wp=(f)$ be a prime ideal of $R$ generated by a not homogeneous element. Since $\wp^{*}$ is a prime ideal, it follows that $\wp^{*} \neq(0)$ because $(0)$ is not prime if $R$ is not a domain. Hence in this case the condition $\Leftarrow$ ) of the Proposition 1 is not true.

In [2] the following definitions related to the graded ideals of $R$ are given.

Definition 2. Let $R$ be a graded ring. A graded ideal $m$ of $R$ is called * maximal if every graded ideal that properly contains $m$ equals $R$.

Definition 3. A graded ring $R$ is a *local ring if it has a unique *maximal ideal.

Example 3. Let $R$ be a graded ring and $\wp$ be a prime ideal of $R$. Let $S$ be the set of the homogeneous elements of $R$ that don't lie in $\wp . S$ is a multiplicatively closed set. Denote $R_{(\wp)}$ the homogeneous localization of $R . R_{(\wp)}$ is a graded ring and the graduation is defined setting $\left(R_{(\wp)}\right)_{i}=\left\{\frac{x}{a} \in R_{(\wp)} \mid \operatorname{deg}\left(\frac{x}{a}\right)=i\right\}$, where $\operatorname{deg}\left(\frac{x}{a}\right)=$ $\operatorname{deg}(x)-\operatorname{deg}(a)$, with $x$ and $a$ homogeneous elements. Then $R_{(\wp)}$ is a *local ring with * maximal ideal $\wp^{*} R_{(\wp)}$.

Now we introduce the following definitions.

Definition 4. Let $R$ be a graded ring. A sequence $x_{1}, \ldots, x_{n}$ of homogeneous elements of $R$ is called *regular sequence on $R$ (or a homogeneous $R$-sequence) if the following conditions are satisfied:

1) $\left(x_{1}, \ldots, x_{n}\right)$ is a proper homogeneous ideal;

2) $x_{i+1}$ is a regular homogeneous element in $R /\left(x_{1}, \ldots, x_{i}\right)$, for $i=0, \ldots, n-1$.

Definition 5. Let $R$ be a graded ring and $E$ be a finitely generated graded $R$ module. A sequence $x=\left\{x_{1}, \ldots, x_{n}\right\}$ of homogeneous elements of $R$ is called $E$ ${ }^{*}$ regular sequence (or a homogeneous $E$-sequence) if the following conditions are satisfied:

1) $\underline{x} E \neq E$

2) $x_{i}$ is a nonzero-divisor in $E /\left(x_{1}, \ldots, x_{i-1}\right) E$, for $i=1, \ldots, n$.

Definition 6. Let $R$ be a graded ring, $E$ be a finitely generated graded $R$-module and $I$ be an ideal of $R$ such that $I E \neq E$. The common length of the maximal homogeneous $E$-sequences in $I^{*}$ is called the ${ }^{*}$ grade of $I$ on $E$, denoted by ${ }^{*} \operatorname{grade}(I, E)$.

A special situation occurs for *local rings: 
Definition 7. Let $(R, m)$ be a graded noetherian ring and $E$ be a finitely generated graded $R$-module. The ${ }^{*}$ grade of $m$ on $E$ is called the ${ }^{*}$ depth of $E$, denoted by *depth $(E)$.

Let $I$ be an ideal of a graded ring $R$. It is customary to set: ${ }^{*} \operatorname{grade}(I)={ }^{*} \operatorname{grade}(R / I)$ $={ }^{*} \operatorname{grade}(I, R)$. More precisely we give the following:

Definition 8. The * grade of $I$ is the common length of the maximal homogeneous $R$-sequences in $I^{*}$, denoted ${ }^{*} \operatorname{grade}(I)$.

Definition 9. $I$ is a * complete intersection if it can generated by a number of homogeneous elements equal to its * grade.

\section{2. ${ }^{*}$ COMPLETE INTERSECTIONS}

Let $R$ be a graded ring.

Definition 10. Let $A=\left(a_{j i}\right)$ be a $m \times n$ matrix whose entries are homogeneous elements of $R$, for $i=1, \ldots, n, j=1, \ldots, m$. $A$ is called a graded matrix on $R$.

Definition 11. Let $A=\left(a_{j i}\right)$ be a graded $m \times n$ matrix on $R$ for $i=1, \ldots, n$, $j=1, \ldots, m$. The ideal of $R$ generated by the $t \times t$ minors of $A, 1 \leq t \leq \min (m, n)$ is called $t$-determinantal ideal, denoted $I_{t}(A)$.

By definition $I_{0}(A)=R$ and $I_{t}(A)=0$ for $t>\min (m, n)$.

Remark 3.

1) If the entries of the matrix $A$ are homogenous elements of the same degree, $I_{t}(A)$ is a graded ideal.

2) If the elements of every column (row) are homogenous of the same degree, then the ideal $I_{t}(A)$ is graded.

Remark 4. If the matrix $A$ is associated to a graded homomorphism $\varphi$ of free graded modules, then $I_{t}(\varphi)$ denotes the ideal $I_{t}(A)$.

Theorem 1. The homogeneous linear forms of $R\left[Y_{1}, \ldots, Y_{m}\right]$

$$
a_{i}=\sum_{j=1}^{m} a_{j i} Y_{j}, 1 \leq i \leq n,
$$

generate $a^{*}$ complete intersection of ${ }^{*}$ grade $n$ if and only if the determinantal ideals of $A=\left(a_{j i}\right)$ satisfy the conditions

$$
{ }^{*} \operatorname{grade}\left(I_{k}(A)\right) \geq n-k+1,1 \leq k \leq n,
$$

where $A$ is a graded matrix whose entries are homogeneous elements of $R$ of the same degree $d_{i}$ for each column. 
Proof. Set $S=R\left[Y_{1}, \ldots, Y_{m}\right]$. Let $a_{i}=\sum_{j=1}^{m} a_{j i} Y_{j} \in S$.

Being $\left(a_{1}, \ldots, a_{n}\right) \subset\left(Y_{1}, \ldots, Y_{m}\right)$ and $\operatorname{grade}\left(Y_{1}, \ldots, Y_{m}\right)=m$, then we suppose $n \leq m$.

$\Rightarrow)$ Let $\left(a_{1}, \ldots, a_{n}\right)$ be a * complete intersection of * grade $n$ in $R\left[Y_{1}, \ldots, Y_{m}\right]$ with $a_{i}$ homogeneous linear forms in the variables $Y_{j}$ and bidegree $\left(g_{i}, 1\right)$. We consider the Koszul complex on $a_{1}, \ldots, a_{n}$ that is a graded complex. By [1] (Proposition 1) the assert follows.

$\Leftarrow)$ We use induction on $n$ and we prove that $a_{1}, \ldots, a_{n}$ generate a ${ }^{*}$ complete intersection in $S$ of * grade $n$.

$n=1$ : we prove that $a_{1}$ is a nonzero-divisor if and only if $* \operatorname{grade}\left(a_{11}, a_{21}, \ldots, a_{m 1}\right) \geq$ 1. If $*$ grade $\left(a_{11}, a_{21}, \ldots, a_{m 1}\right) \geq 1$, then some homogenous element $a_{j 1}$ is nonzerodivisor. It follows that $a_{j 1} Y_{j}$ is a nonzero-divisor. Choose an element $f \in S$ with $f \neq 0$, one has

$$
f a_{1}=f\left(a_{11} Y_{1}+a_{21} Y_{2}+\cdots+a_{m 1} Y_{m}\right) \neq 0
$$

because at least $f a_{j 1} Y_{j} \neq 0$. Then $a_{1}$ is a nonzero-divisor. Conversely, suppose $a_{1}$ nonzero-divisor. For all nonzero element $f \in S$, one has $f a_{1} \neq 0$. It follows

$$
f a_{1}=f\left(a_{11} Y_{1}+a_{21} Y_{2}+\cdots+a_{m 1} Y_{m}\right) \neq 0
$$

hence $f a_{j 1} Y_{j} \neq 0$ for all $f$ and for any $j=1, \ldots, m$. Being $Y_{j}$ nonzero-divisor one has that each $a_{j 1}$ is nonzero-divisor and ${ }^{*} \operatorname{grade}\left(a_{11}, a_{21}, \ldots, a_{m 1}\right) \geq 1$.

Suppose by induction that the assert is true for $n-1$ homogeneous elements. Let $\wp$ be a graded prime ideal of $S$ associated to a maximal homogeneous $S$-regular sequence in $\left(a_{1}, \ldots, a_{n}\right)$ and containing $a_{i}$ 's. Hence:

$$
{ }^{*} \operatorname{grade}(\wp)={ }^{*} \operatorname{grade}\left(\wp S_{\wp}\right)={ }^{*} \operatorname{grade}\left(a_{1}, \ldots, a_{n}\right) S_{\wp} .
$$

It is sufficient to prove that ${ }^{*} \operatorname{grade}(\wp)$ can not be smaller than $n$. In fact from ${ }^{*} \operatorname{grade}(\wp) \geq n$ it follows that ${ }^{*} \operatorname{grade}(\wp)=n$. Assume that ${ }^{*} \operatorname{grade}(\wp)<n$. By hypothesis for per $k=1$ one has ${ }^{*} \operatorname{grade}\left(I_{1}(A)\right) \geq n$, then some $a_{j i}=a$ is not in $\wp$. Consider the ring $R_{(a)}$, that is the homogeneous localization of $R$ in the set of powers of $a$, with $a \notin \wp . R_{(a)}$ is a *local ring, whose graduation is given by $\left(R_{(a)}\right)_{i}=\left\{\frac{x}{y} \in\right.$ $\left.R_{(a)} \mid \operatorname{deg}\left(\frac{x}{y}\right)=i\right\}$, where $\operatorname{deg}\left(\frac{x}{y}\right)=\operatorname{deg}(x)-\operatorname{deg}(y)$ with $x$ and $y$ homogeneous elements of $R$ (Example 3). Consider the graded ideal $J=\left(a_{1}, \ldots, a_{n}\right)_{(a)}$ of $R_{(a)}$ and set a system of generators consisting of homogenous linear forms with coefficient in $R_{(a)}$. By elementary transformations over $R_{(a)}$ of the matrix $A$, which involve a linear change of the indeterminates $Y_{1}, \ldots, Y_{m}$ to $T_{1}, \ldots, T_{m}$, we can assume that $J$ is generated by $T_{1}$ and $b_{2}, \ldots, b_{m}$, with $b_{i}=\sum_{j=2}^{m} b_{j i} T_{j} \in R_{(a)}\left[T_{2}, \ldots, T_{m}\right]$ and $b_{j i} \in R(a)$. We obtain a graded matrix $B=\left(b_{j i}\right)$ whose entries are homogenous elements of the same degree in each column. Hence

$$
I_{k}(A)_{(a)}=I_{k-1}(B),
$$


with $I_{k}(A)_{(a)}$ graded ideal of $R_{(a)}$ obtained by a homogeneous localization of the graded ideal $I_{k}(A)$. Then:

$$
{ }^{*} \operatorname{grade}\left(I_{k-1}(B)\right)={ }^{*} \operatorname{grade}\left(I_{k}(A)_{(a)}\right) \geq{ }^{*} \operatorname{grade}\left(I_{k}(A)\right) \geq n-k+1,
$$

hence * $\operatorname{grade}\left(I_{k-1}(B)\right) \geq(n-1)-(k-1)+1$.

By induction hypothesis one concludes that $b_{2}, \ldots, b_{m}$ generate a * complete intersection of * grade $n-1$ in $R(a)\left[T_{2}, \ldots, T_{m}\right]$. It is enough to prove that $T_{1}, b_{2}, \ldots, b_{m}$ generate a *omplete intersection of grade $n$ in $R(a)\left[T_{2}, \ldots, T_{m}\right]$. Being $T_{1}$ a nonzerodivisor in $R(a)\left[T_{2}, \ldots, T_{m}\right]$, it follows that $T_{1}, b_{2}, \ldots, b_{m}$ generate a *omplete intersection. It follows ${ }^{*} \operatorname{grade}\left(a_{1}, \ldots, a_{n}\right)_{(a)}=n$, hence

$$
{ }^{*} \operatorname{grade}\left(a_{1}, \ldots, a_{n}\right) S_{\wp} \geq n \text {. }
$$

Being

$$
{ }^{*} \operatorname{grade}(\wp)={ }^{*} \operatorname{grade}\left(a_{1}, \ldots, a_{n}\right) S_{\wp}
$$

one has $* \operatorname{grade}(\wp) \geq n$, that is a contradiction.

Now, let $R=K\left[X_{1}, \ldots, X_{q}\right]$ be the polynomial ring over a field $K$ with standard graduation. Let $E=R f_{1}+\cdots+R f_{m}$ be a finitely generated graded $R$-module, $d_{j}=\operatorname{deg}\left(f_{j}\right)$ for $j=1, \ldots, m$, and $\operatorname{Sym}_{\mathrm{R}}(E)=\bigoplus_{t \geq 0} \operatorname{Sym}_{\mathrm{t}}(E)$ be its symmetric algebra.

If $E$ has free graded presentation:

$$
\bigoplus_{i=1}^{n} R\left(-c_{i}\right) \stackrel{\varphi}{\rightarrow} \bigoplus_{j=1}^{m} R\left(-d_{j}\right) \stackrel{\psi}{\rightarrow} E \rightarrow 0,
$$

with $\varphi=\left(a_{j i}\right)$ graded matrix on $R$, then the kernel $J$ of the surjective homomorphism $\operatorname{Sym}(\psi)$ induced by $\psi$

$$
\operatorname{Sym}(\psi): R\left[Y_{1}, \ldots, Y_{m}\right] \rightarrow \operatorname{Sym}_{R}(E) \rightarrow 0
$$

is generated by linear forms in the variables $Y_{j}$

$$
a_{i}=\sum_{j=1}^{m} a_{j i} Y_{j}
$$

for $i=1, \ldots, n$ and such that $\sum_{j=1}^{m} a_{j i} f_{j}=0,1 \leq i \leq n$.

Hence the symmetric algebra $\operatorname{Sym}_{R}(E)$ has a presentation $R\left[Y_{1}, \ldots, Y_{m}\right] / J$. The ring $R\left[Y_{1}, \ldots, Y_{m}\right]=K\left[X_{1}, \ldots, X_{q} ; Y_{1}, \ldots, Y_{m}\right]$ is bigraded by $\operatorname{bideg}\left(X_{i}\right)=(1,0)$ for $i=1, \ldots, q$ and $\operatorname{bideg}\left(Y_{j}\right)=\left(0, d_{j}\right)$ for $j=1, \ldots, m$.

Remark 5. If $E$ is a free graded $R$-module of rank $m$, then its symmetric algebra is

with $E=\bigoplus_{j} R^{m}\left(-d_{j}\right)$.

$$
\operatorname{Sym}_{R}(E) \cong R\left[Y_{1}, \ldots, Y_{m}\right],
$$

By Theorem 1 it follows the following result for $\operatorname{Sym}_{R}(E)$. 
Corollary 1. Let $E=R f_{1}+\cdots+R f_{m}$ be a finitely generated graded module on $R=K\left[X_{1}, \ldots, X_{q}\right]$, with $d_{j}=\operatorname{deg}\left(f_{j}\right)$ for $j=1, \ldots$, m. Let

$$
F_{1} \stackrel{\varphi}{\rightarrow} F_{0} \stackrel{\psi}{\rightarrow} E \rightarrow 0
$$

be a free graded presentation of $E$ with $F_{0}$ and $F_{1}$ free graded modules of rank $n$ and $m$, respectively, and $\varphi=\left(a_{j i}\right)$ be a graded matrix whose columns have homogenous elements of $R$ of the same degree. The kernel of the canonical epimorphism

$$
\operatorname{Sym}(\psi): R\left[Y_{1}, \ldots, Y_{m}\right] \rightarrow \operatorname{Sym}_{R}(E) \rightarrow 0
$$

is a ${ }^{*}$ complete intersection of ${ }^{*}$ grade $n$ in $R\left[Y_{1}, \ldots, Y_{m}\right]$ if and only if

$$
{ }^{*} \operatorname{grade}\left(I_{k}(\varphi)\right) \geq n-k+1,1 \leq k \leq n \text {. }
$$

\section{REFERENCES}

[1] L. L. Avramov, "Complete intersections and symmetric algebras,” J. Algebra, vol. 73, pp. 248-263, 1981.

[2] W. Bruns and J. Herzog, Cohen-Macaulay rings, ser. Cambridge Studies in Advanced Mathematics. Cambridge: Cambridge University Press, 1998, vol. 39.

[3] M. La Barbiera, "Conditions $\left(S_{q}\right)$ and $\left(G_{q}\right)$ on graded rings," Atti Accad. Peloritana Pericolanti, Cl. Sci. Fis. Mat. Nat., vol. 84, pp. c1a0 601 007, 9, 2006.

[4] M. La Barbiera, "On graded ${ }^{*} G_{q}$-rings," Int. Math. Forum, vol. 5, no. 1-4, pp. 191-198, 2010.

Author's address

\section{M. la Barbiera}

University of Messina, Department of Mathematics, Viale Ferdinando Stagno d'Alcontres, 31, 98166 Messina, Italy

E-mail address: monicalb@unime.it 